\title{
Controle das parasitoses gastrintestinais em ovinos e caprinos na região semiárida do Nordeste do Brasil ${ }^{1}$
}

\author{
Valéria M.M. Costa ${ }^{2}$, Sara V.D. Simões ${ }^{2}$ e Franklin Riet-Correa ${ }^{2}$
}

\begin{abstract}
Costa V.M.M., Simões S.V.D \& Riet-Correa F. 2011. [Gastro-intestinal nematodes control in goats and sheep in the semiarid region northeastern Brazil.] Controle das parasitoses gastrintestinais em ovinos e caprinos na região semiárida do Nordeste do Brasil. Pesquisa Veterinária Brasileira 31(1):65-71. Hospital Veterinário, Centro de Saúde e Tecnologia Rural, Universidade Federal de Campina Grande, Patos, PB 58700-970, Brazil. E-mail: valery.medeiros@hotmail.com

This paper reviews some important concepts to be considered for the control of gastrointestinal nematode parasites of sheep and goats, including the epidemiology of this parasitic disease in the Brazilian semiarid, and the occurrence of anti-helminthic resistance in the region. Considering the widespread use of preventive treatments during the dry season, new alternatives for the control of gastrointestinal parasites are recommended, taking into account the parasite integrated control system and the use of methods to allow the presence of susceptible parasites in the refugia. Differences between sheep and goats are discussed, taking into account the higher susceptibility of goats and the necessity to use larger doses in goats than in sheep for most anti-helminthics.
\end{abstract}

INDEX TERMS: Anti-helminthics, gastrointestinal nematodes, anti-helminthic resistance, integrated parasitic control, semiarid.

RESUMO.- Neste artigo são revisados alguns conceitos importantes a serem considerados para o controle de parasitas gastrintestinais em caprinos e ovinos. Descrevem-se as características epidemiológicas desta parasitose no semiárido e o aparecimento de resistência aos anti-helmínticos na região. São propostas alternativas para o controle da doença levando em consideração, principalmente, a adoção de técnicas de controle integrado de parasitas com uma mudança nos sistemas de tratamento preventivo para sistemas que permitam a sobrevivência de parasitas susceptíveis na refugia. São discutidas as diferenças entre caprinos e ovinos, considerando que os caprinos são mais susceptíveis e que para maioria das drogas devem ser tratados com doses maiores.

TERMOS DE INDEXAÇÃO: Anti-helmínticos, nematódeos gastrintestinais, resistência anti-helmíntica, controle integrado de parasitas, semiárido.

\section{INTRODUÇÃO}

O Brasil detém 3,3 \% da população mundial de caprinos e ovinos, estimada em 9,5 milhões e 14,6 milhões, respecti-

\footnotetext{
${ }^{1}$ Recebido em 24 de abril de 2010.

Aceito para publicação em 17 de agosto de 2010.

2 Hospital Veterinário, Centro de Saúde e Tecnologia Rural (CSTR), Universidade Federal de Campina Grande, Campus de Patos, 58700-970 Patos, PB, Brasil. *Autor para correspondência: valery.medeiros@ @otmail.com
}

vamente. A região Nordeste concentra os maiores rebanhos, com $94 \%$ dos caprinos e $55 \%$ dos ovinos (Sebrae 2009). No entanto, um dos principais entraves ao crescimento deste segmento são as parasitoses por nematódeos gastrintestinais, que representam o maior e mais grave problema sanitário dos pequenos ruminantes, chegando a inviabilizar economicamente a criação (Vieira 2008).

Neste trabalho são revisados alguns conceitos que devem ser considerados para o controle das parasitoses gastrointestinais. São discutidos os principais gêneros de parasitas que acometem caprinos e ovinos no Nordeste, os principais fatores epidemiológicos para a região, a resistência aos anti-helmínticos e algumas das medidas de controle e tratamento, incluindo métodos alternativos que visam, principalmente, diminuir a contaminação dos animais e da pastagem, manter a eficácia das drogas antiparasitárias e reduzir os prejuízos econômicos causados pela verminose.

\section{CONCEITOS PARA O CONTROLE DE HELMINTOS GASTRINTESTINAIS}

\section{Refugia}

O termo refugia é utilizado para definir um grupo de larvas que permanece na pastagem sem sofrer ação das drogas, sendo consideradas como um estoque de larvas 
susceptíveis. Os parasitas adultos que estão dentro dos animais e que não recebem tratamento químico são, também, uma população de refugia. Um fator importante para minimizar o fenômeno da resistência antihemíntica (RA) são as larvas em refugia, pois o aparecimento da RA está intimamente ligado ao sucesso da progênie que sobreviveu ao tratamento (Van Wyk 2001). As larvas em refugia permanecem com seu caráter susceptível, pois ficam livres de qualquer medida de seleção para RA (Molento 2005), contribuindo para a diluição dos genes que codificam para RA nas próximas gerações (Van Wyk 2001). O tamanho da população em refugia tem papel fundamental na manutenção da eficácia das drogas, retardando o processo de seleção.

\section{Resistência e resiliência dos hospedeiros}

Resistência é a capacidade do hospedeiro de impedir o desenvolvimento de parasitos, podendo diminuir o estabelecimento das $L_{3}$ retardar o crescimento dos parasitas, reduzir a produçẫo de ovos ou eliminar os parasitas existentes (Torres-Acosta \& Hoste 2008). Resiliência é a capacidade do hospedeiro de resistir à infecção parasitária. Como os parasitas se instalam no sistema digestivo os animais resilientes, ao contrário dos resistentes, não diminuem significativamente a contaminação ambiental (Torres-Acosta \& Hoste 2008).

Embora as mesmas espécies de nematódeos ocorram em ovelhas e cabras, a infecção parasitária difere largamente entre os dois hospedeiros, de acordo com sua imunidade, fisiologia e características comportamentais. Em condições de pastoreio, em pastagens, os caprinos são mais infectados do que os ovinos, sendo isto relacionado, provavelmente, à sua menor habilidade para desenvolver uma resposta imune contra os nematódeos (Hoste et al. 2007). Consequentemente, em condições de pastoreio, cabras, mesmo adultas, devem ser consideradas particularmente de risco (Torres-Acosta \& Hoste 2008). Em contraste, em condições de consumo de foIhas e ramas de árvores ou arbustos (ramoneio) em áreas de caatinga, as ovelhas são usualmente mais infectadas do que as cabras, por causa da vantagem da atitude comportamental destas últimas para explorar brotos em posição bipedal, evitando contato excessivo com os estágios infectantes (Vercruysse 1983, Hoste et al. 2007). $\mathrm{O}$ consumo de folhas e ramas de árvores ou arbustos em posição bipedal ocorre também com as ovelhas deslanadas do Nordeste, mas com menor freqüência do que em caprinos.

Os animais jovens são mais susceptíveis do que os adultos, que são menos predispostos devido à imunidade estabelecida pelas infecções anteriores (Girão et al. 1980, Ahid et al. 2008).

Uma boa nutrição energética e protéica aumenta a resistência dos animais às infecções parasitárias, portanto, sempre que for viável economicamente, a correta suplementação do rebanho diminui o grau de infecção. Por outro lado períodos de carência alimentar aumentam a susceptibilidade aos parasitos e favorecem a ocorrência de sinais clínicos (Torres-Acosta \& Hoste 2008).

\section{Hipobiose}

Hipobiose ou desenvolvimento larval inibido é um artifício usado pelos parasitas para evitar condições climáticas adversas às suas progênies e permanecer sexualmente imaturos até que haja boas condições para seu desenvolvimento. Sua importância epidemiológica se deve ao fato de assegurar a sobrevivência do nematódeo, no hospedeiro, durante períodos adversos. A subseqüente maturação de larvas inibidas aumenta a contaminação do meio ambiente, podendo, às vezes, resultar em doença clínica. $\mathrm{O}$ acúmulo de larvas hipobióticas coincide com o início do período seco nas regiões tropicais e subtropicais (Vieira 1997). Sua volta ao estado larval maturo coincide com o retorno das condições favoráveis no início do período chuvoso, no entanto, não está claro o que dispara o sinal para a volta da maturação dessas larvas (Urquhart 1998).

\section{Fenômeno do aumento da eliminação de ovos no peri- parto}

É o aumento da quantidade de ovos de parasitas gastrintestinais eliminado por ovelhas e cabras no período do peri-parto (spring rise, periparturient rise ou queda da imunidade do periparto). Os mecanismos pelos quais o fenômeno ocorre ainda são desconhecidos, porém acredita-se que sejam provocados por imunossupressão de origem endócrina, decorrente de variações hormonais que ocorrem próximas ao parto e durante a lactação. Essa queda na imunidade permite o desenvolvimento de larvas em hipobiose e/ou um maior estabelecimento de novas larvas, ou, ainda, uma maior fecundidade de adultos existentes, o que resulta em aumento no número de ovos eliminados nas fezes (Stear et al. 1997). A ocorrência desse fenômeno, numa época em que as quantidades de novos hospedeiros susceptíveis estão aumentados, garante a sobrevivência e a propagação da espécie do verme.

\section{Resistência anti-helmíntica}

A resistência anti-helmíntica $(R A)$ é definida como a capacidade de uma população de parasitas em sobreviver a doses de anti-helmínticos que poderiam ser letais para populações susceptíveis (Vieira 2008, Torres-Acosta \& Hoste 2008). Esta habilidade de sobreviver a futuras exposições de uma droga pode ser transmitida aos seus descendentes. Este fenômeno ocorre frente a todos os compostos químicos com graves conseqüências econômicas no mundo todo. Quando a RA ocorre entre produtos do mesmo grupo químico é chamada de lateral. Quando são envolvidas duas drogas, de grupos diferentes, este fenômeno é chamado de RA cruzada. A resistência antihelmíntica múltipla (RAM) ocorre quando um parasita é resistente a mais de duas bases farmacológicas (Molento 2005).

Geralmente, suspeita-se de RA quando se obtém uma baixa resposta após um tratamento anti-helmíntico. Por 
outro lado, uma falha na resposta ao vermífugo não significa, necessariamente, um caso de RA, pois alguns sinais clínicos não são específicos e podem ser devido a outras doenças ou a nutrição deficiente (Vieira 2008).

A RA é diagnosticada, na maioria dos casos, após a observação empírica da pouca eficácia da medicação utilizada, sendo um dos maiores entraves a falta de métodos sensíveis para quantificá-la (Molento 2005). O método mais simples para determinar a RA é mediante a contagem de ovos nas fezes após o tratamento, embora o resultado deste teste não seja consistente, pois pode apresentar variações acima de $20 \%$. Quando após a aplicação do produto a eficiência do mesmo for inferior a $95 \%$, deve-se fazer um teste de RA que é importante para escolher a droga a ser utilizada. Para isso é necessário administrar drogas de diferentes grupos químicos (benzimidazóis, lactonas macrocíclicas, levamisol, closantel e se necessário triclorfon) a grupos de 10 animais, incluindo um grupo controle. A diminuição no número de OPG indica a eficiência de cada droga e com este resultado deve ser escolhida a droga a ser utilizada. O Grupo Mercado Comum (1996) avalia a atividade dos antiparasitários da seguinte forma: altamente efetivo quando reduz mais que $98 \%$; efetivo quando reduz 90-98\%; moderadamente efetivo $80-89 \%$ e insuficientemente ativo menos que $80 \%$.

No nordeste brasileiro, suspeitou-se de RA em nematodeos de caprinos no Ceará (Vieira et al. 1989). Estudos posteriores indicaram RA, em caprinos, à levamisol, albendazol e parbendazol em Pernambuco, à albendazol e ivermectina na Bahia (Charles et al. 1989, Barreto \& Silva 1999), e à oxfendazol, levamisol, closantel e ivermectina no Ceará (Melo et al. 2003, Vieira \& Cavalcante 1999). Em ovinos foi detectada RA à oxfendazole no Ceará (Melo et al. 2003).

\section{Formas de tratamento com anti-helmínticos}

Tratamento preventivo é realizado em períodos regulares, em datas pré-estabelecidas, em todo o rebanho, com o objetivo de evitar infecções clínicas ou subclínicas. No entanto, o princípio ativo não elimina $100 \%$ das formas infectantes, selecionando cepas resistentes (Molento 2005). Tratamento curativo é realizado somente quando ocorrem sinais clínicos evidentes. Tratamento tático é utilizado sempre que as condições ambientais favoreçam o surgimento de verminose. Tanto no tratamento curativo quanto no tático o desenvolvimento da RA é retardado, mas existe uma importante perda de produção pelos animais além de alta contaminação do meio-ambiente com os estágios infectantes dos nematódeos. Tratamento supressivo consta em vermifugar os animais a cada 2-4 semanas, com drogas de curta persistência. A razão disto é empregar drogas antes do fim do período pré-patente dos parasitas, portanto objetivando uma quase total eliminação de vermes no ambiente (Reis 2007, Torres-Acosta \& Hoste 2008). Embora seja eficiente no controle do parasitismo, precipita o rápido aparecimento da RA. Tratamento seletivo é quando se tratam somente alguns animais do rebanho. Tratamento não intencional é quando se utiliza anti-helmínticos para o tratamento de outras doenças parasitárias como a miíase por Oestrus ovis ou a sarna (Torres-Acosta \& Hoste 2008).

\section{Controle integrado de parasitas}

O controle integrado de parasitas (CIP) é definido como a adoção de um conjunto de medidas estratégicas que visam principalmente reduzir a contaminação dos animais e da pastagem, assim como manter a eficácia das drogas antiparasitárias. O CIP deve ser utilizado em todas as situações, mas, particularmente quando existe RA. Requer componentes importantes, como a disponibilidade de técnicas para o diagnóstico de RA, verificação da eficiência dos anti-helmínticos, conhecimento da epidemiologia parasitária local e uma troca na mentalidade de técnicos e produtores para utilizar métodos menos dependentes dos anti-helmínticos. A dependência de anti-helmínticos comerciais no controle dos nematódeos gastrintestinais tem demonstrado ser pouco sustentável e eficiente a longo prazo (Molento 2005).

Recentemente foram revisadas algumas das técnicas alternativas a serem utilizadas, no Brasil, para o CIP, incluindo o manejo do rebanho e de pastagens, pastoreio rotacionado, descontaminação prévia das pastagens, pastoreio com alternância de categorias e ou espécies de hospedeiros, controle biológico, seleção genética, nutrição, vacinas e fitoterapia (Cezar et al. 2008).

\section{Método Famacha}

É a técnica mais conhecida de tratamento seletivo, uma vez que são vermifugados apenas os animais que apresentam anemia clínica (Molento 2005). Além disso, permite identificar animais susceptíveis, resilientes e resistentes, proporcionando informações para um programa de seleção. No momento da avaliação, se define a coloração da conjuntiva frente a um cartão ilustrativo que acompanha a técnica e se determina o grau de anemia dos animais. Neste cartão, estão presentes cinco categorias, variando de 1 (coloração vermelho brilhante) até 5 (coloração pálida, quase branco), que representam diferentes valores de hematócrito, sendo $35,25,20,15$ e $10 \%$, respectivamente, para os grupos de 1 a 5 (Bath et al. 2001, Van Wyk 2002). Baseado nesta comparação são tratados somente os animais que apresentam coloração de mucosas compatíveis com os graus 4 e 5 e, em alguns casos, com o grau 3. Este procedimento permite que haja persistência de uma população de parasitas sensível no meio ambiente, mantém a eficácia anti-helmíntica por um período maior e com isso, o aparecimento de RA tende a ser retardado (Vieira 2008). Em adição, o método, proporciona uma economia média de $58,4 \%$ nos custos com a aquisição de anti-helmínticos (Bath \& Van Wyk et al. 2001).

No Brasil, trabalhos realizados no Paraná e Rio Grande do Sul comprovaram a eficiência do método, diminuindo o número de tratamentos com anti-helmínticos e mantendo a eficácia dos produtos (Molento et al. 2004). Estudos 
realizados no Nordeste em caprinos comprovaram a viabilidade do Método Famacha nessa espécie, no entanto a coloração da conjuntiva de caprinos sadios tem menor intensidade quando comparada com ovinos sadios e o preenchimento capilar nos caprinos é mais demorado que em ovinos, devendo ser observada a mucosa por pelo menos oito segundos após sua exposição, o que na espécie ovina é realizada de imediato (Reis 2004). Além disso, no início do experimento Hemonchus spp. foi o parasito mais freqüente, enquanto que após um período de um ano, houve uma predominância de Trichostrongylus spp., que não é controlado pelo Método Famacha (Reis 2004).

\section{SITUAÇÃO DO NORDESTE}

\section{Principais gêneros de parasitas}

Os caprinos e ovinos criados na região semiárida são parasitados por Haemconchus contortuse Trichostrongylus axei, que se localizam no abomaso, Trichostrongylus colubriformis, Strongyloides papillosus, Cooperia punctata, Cooperia pectinata e Bunostomum trigonocephalum, que parasitam o intestino delgado, e Oesophagostomum colubianum, Trichuris ovis, Trichuris globulosa e Skrjabinema sp., que vivem no intestino grosso. Haemconchus contortus, Trichostrongylus colubriformis, Strongyloides papillosus e Oesophagostomum colubianum são os que apresentam maior prevalência e maior intensidade de infecção, sendo considerados os nematódeos de maior importância econômica para e exploração de caprinos e ovinos no Nordeste. Na região semiárida da Paraíba $H$. contortus foi o parasita mais prevalente do abomaso e as maiores infecções ocorreram nos meses de fevereiro, junho e dezembro. S. papillosus e C. curticei prevalecem no intestino delgado em fevereiro, maio e junho e $O$. columbianum e T. globulosa, no intestino grosso em março, maio e julho; estas espécies estão presentes no decorrer de todo o ano, apesar das variações climáticas (Costa \& Vieira 1984, Silva et al. 1998). Levantamentos realizados revelam que mais de $80 \%$ da carga parasitária de caprinos é composta por H. contortus (Costa \& Vieira 1984, Girão et al. 1992, Arosemena et al. 1999).

Martins Filho \& Menezes (2001) encontraram em caprinos do estado da Paraíba, larvas do gênero Oesophagostomum sp., Cooperia sp., Haemonchus sp., Trichostrongylus sp. e Bunostomum sp. em 63,33\% das amostras pesquisadas. Também foram encontrados ovos de Strongyloides sp. (57,47\%), Trichuris sp. (7,43\%) e Toxocara sp. $(0,82 \%)$. Identificaram larvas de terceiro estádio de Oesophagostomum sp. (46\%), Cooperia sp. (30\%), Haemonchus sp. (10\%), Trichostrongylus sp. (12\%) e Bunostomum sp. (0,2\%). Pereira (1976), em Pernambuco, em caprinos na raça Moxotó, demonstrou a ocorrência de $H$. contortus, T. colubriformis, O. columbianum, S. ovis e Cysticercus tenuicollis. A prevalência entre os grupos estudados foi maior no final do inverno (junho, julho e agosto) e o menor no final da estação seca (novembro e dezembro). No Ceará, O. columbianum foi à espécie que se apresentou com maior intensidade e freqüência du- rante os meses de maio a agosto, estando ausente nos demais meses nos quais pouca ou nenhuma precipitação foi registrada (Arosemena et al. 1999). Nesse mesmo trabalho foi verificado um aumento da ocorrência $S$. papillosus a partir de fevereiro, associado ao aumento da pluviosidade, imprescindível para a sobrevivência desta espécie.

\section{Características da Região e aspectos epidemiológicos das parasitoses gastrintestinais}

A região semiárida do Nordeste apresenta clima semiárido quente com chuvas de $300-800 \mathrm{~mm}$ anuais. As chuvas se caracterizam por serem irregulares, ocorrendo anos sem chuvas significativas ou com poucas chuvas. O período de chuvas é curto, de 3-4 meses e, dependendo da região, se estende de janeiro-fevereiro a abril-maio ou de março-abril a maio-junho. A temperatura média é de 26$28^{\circ} \mathrm{C}$, diminuindo um pouco nas áreas mais altas, onde as chuvas são, também, mais abundantes. A umidade é bai$x a$, variando de $60 \%$ a $75 \%$ com alta evapotranspiração e radiações solares. A vegetação, denominada caatinga (do Tupi-Guarani: caa [mata] + tinga [branca] = mata branca) é o único bioma exclusivamente brasileiro e ocupa quase $11 \%$ do território nacional. Caracteriza-se por ser adaptada às condições de aridez (xerófila) com forte presença de arbustos com galhos retorcidos e com raízes profundas e presença de cactos e bromélias. Os arbustos perdem, quase que totalmente, as folhas em épocas de seca. Nestas condições a precipitação é o fator climático mais importante no aparecimento das infecções por nematódeos gastrintestinais nos rebanhos (Vieira 2007). De janeiro a maio ocorrem precipitações, com média mensal superior a $50 \mathrm{~mm}$. Esse aspecto é de fundamental importância na epidemiologia, visto que a transmissão da maioria dos nematódeos só ocorre com esse mínimo de precipitação (Costa \& Vieira 1984). Por outro lado, na época da seca os parasitas não sobrevivem no meio ambiente, permanecendo durante esse tempo nos hospedeiros (Vieira 2007). No semiárido do Nordeste, assim com em outras regiões semiáridas, a sobrevivência das larvas no meio ambiente é consideravelmente menor do que em regiões temperadas variando de 30 a 90 dias (Vieira 1997, Torres-Acosta \& Hoste 2008).

Um fato importante que tem levado ao aumento das infecções por parasitas gastrointestinais no semiárido é o recente incremento da ovinocaprinocultura com adoção de técnicas semi-intensivas de criação. Em áreas de criação extensiva, onde caprinos e ovinos são mantidos exclusivamente na caatinga, com baixas lotações, de um animal por hectare ou a cada dois hectares, as infecções por parasitas gastrintestinais são raras. Com a aplicação de técnicas semi-intensivas de produção, com introdução de pastagens cultivadas, que proporcionam sombreamento, evitando a dessecação de ovos e larvas, e o aumento das lotações, aumentaram significativamente a frequência das helmintoses gastrintestinais dos ovinos e caprinos. Outro fator que pode ter aumentado a frequência da parasitose 
nas criações semi-intensivas é a maior susceptibilidade de caprinos ou ovinos de raças importadas com maior produtividade em relação a raças nativas. Esta situação tem sido evidenciada em caprinos da raça Boer e em ovinos Dorper em relação às raças de caprinos nativas, nas mesmas condições de criação (Valéria M.M. Costa 2008, dados não publicados). Em estudo retrospectivo dos surtos de parasitose gastrintestinal na região semiárida constatou-se que estas parasitoses são a doença mais freqüente de caprinos e ovinos, que o número de casos de verminose foi crescente nos meses de fevereiro a junho, sendo a maior frequência neste último, e que praticamente todos os surtos são causados por Hemonchus spp., evidenciando que qualquer plano de controle parasitário deve objetivar o controle deste parasita (Costa et al. 2009). Nesse trabaIho foi constatado, também, que os caprinos são mais afetados que os ovinos e que em surtos em rebanhos mistos de caprinos e ovinos é freqüente que ocorram sinais clínicos somente nos caprinos (Costa et al. 2009).

\section{PERSPECTIVAS PARA O CONTROLE NO NORDESTE}

Atualmente o controle estratégico mais utilizado no semiárido consiste em medicar o rebanho quando as condições climáticas da região são desfavoráveis ao desenvolvimento e sobrevivência dos estágios de vida livre no ambiente. Para isso realiza-se a aplicação de vermífugos quatro vezes por ano. A primeira medicação do ano em julho ou agosto, no início da seca, a segunda, aproximadamente 60 dias após, a terceira, em novembro (final da seca) e a última em março (meados do período de chuvas) (Vieira et al. 1997). Além disso, medicações anti-helmínticas adicionais (táticas) são recomendadas em determinadas circunstâncias, como por exemplo, em rebanhos que utilizam estação de monta, uma medicação deve ser feita antes do início da cobertura ou inseminação artificial e outra 30 dias antes do início do período de parição. Esta última deve ser efetuada com produtos que atuem sobre nematódeos adultos e larvas hipobióticas (Vieira 2008). No entanto, esses tratamentos estratégicos aumentam os problemas de resistência antihelmíntica (RA), já que favorecem a sobrevivência de parasitas resistentes no animal, enquanto desaparecem os parasitas sensíveis em refugia (Torres-Acosta \& Hoste 2008, Vieira 2008, Costa et al. 2009) e, apesar de proporcionar excelentes resultados no curto prazo, quando utilizadas por período prolongado (mais de 5 anos) toda a população de parasitas, pode se tornar resistente (Vieira 2008).

Além dos tratamentos preventivos, a RA também é favorecida pela prática bastante difundida de tratar os animais e posteriormente colocá-los em campos livres de parasitas. Nestas condições somente os parasitos resistentes que sobreviveram ao tratamento, vão contaminar o meio ambiente. Outro aspecto importante que deve ser levado em consideração é a maior susceptibilidade dos caprinos e a necessidade de se empregar, nesta espécie, doses maiores com a maioria dos anti-helmínticos (Torres-Acosta \& Hoste 2008, Costa et al. 2009).
Diante da real possibilidade de aumentar a RA, apesar de esse esquema ser eficiente ainda na maioria das fazendas, é urgente mudar o mesmo, substituindo-o por outro sistema que favoreça a sobrevivência de helmintos não resistentes na refugia. Alternativamente a este método podem ser implementados métodos de tratamento seletivo, que tratem só os animais mais parasitados, ou tratar animais de diferentes idades, com diferente grau de parasitismo, em épocas diferentes. Um desses métodos é o "Famacha", que já está sendo utilizado no Nordeste (Reis 2004, Vieira 2008), e que permite tratar somente os animais mais parasitados, além de permitir a seleção dos animais resistentes. No entanto, a aplicação do Método Famacha no Nordeste tem restrições, principalmente no referente ao treinamento dos produtores para aplicar o método. Outra restrição é a aplicação do método em caprinos e ovinos, que exige interpretações diferentes (Reis 2004).

Uma outra forma de identificar os animais mais parasitados é pela observação dos sinais clínicos em alguns animais, que pode ser feita por produtores experientes. A principal limitante para a utilização deste método é a possibilidade de confundir os sinais de parasitose gastrintestinal com outras doenças ou com subnutrição. Uma alternativa que vem sendo testada é a de realizar contagem de ovos por grama de fezes (OPG) dos animais e somente tratar quando esta passar de certo número (500 ou 1000 OPG, por exemplo) (Valéria M.M. Costa 2008, dados não publicados). Neste caso são realizados exames a cada dois meses durante a seca e mensalmente durante as chuvas. Resultados de trabalhos recentes demonstraram que não são necessários mais do que 2 ou 3 tratamentos durante a época das chuvas para evitar parasitoses clínicas (Valéria M.M. Costa 2008, dados não publicados). Este esquema, como inicia os tratamentos após o inicio da contaminação das pastagens, mesmo tratando todos os animais do rebanho, permite a sobrevivência de estirpes de parasitas suscetíveis aos anti-helmínticos na refugia.

Uma boa possibilidade no semiárido é a utilização de áreas de caatinga, que pela presença de forrageiras nativas arbóreas ou arbustivas e a utilização de menores lotações, diminuem as possibilidades de infecção parasitária. O cultivo de forrageiras arbustivas para o ramoneio, principalmente como banco de proteínas, como a sabiá (Mimosa caesalpinifolia), leucena (Leucaena leucocaphala) e gliricídia (Gliricidia sepium), também é uma boa alternativa para a diminuição da contaminação ambiental por parasitas. Uma forma de eliminar totalmente a infecção parasitária é o confinamento, que no semiárido pode ser adotado utilizando diversas forrageiras adequadas para corte, como concentrado, ou para a produção de silagem ou feno, já largamente utilizado na região, como a palma (Opuntia ficusindica), algaroba (Prosopis juliflora), maniçoba (Manihot spp.), mandioca (Manihot esculenta), leucena, gliricídia, cunhã (Clitoria ternatea), guandú (Cajanus sabia), feijão bravo (Capparis hastata), melancia forrageira (Citrillus lanatus cv. citroides), capim elefante (Pennisetum purpureum), cana de açúcar, sorgo e outras gramíneas cultiva- 
das em áreas de vazante ou irrigadas. O confinamento é um sistema totalmente eficiente para eliminar as infecções por helmintos gastrintestinais, sempre que a forragem verde utilizada seja cortada em áreas sem pastoreio. No feno e na silagem as larvas $L_{3}$ não sobrevivem (Torres-Acosta \& Hoste 2008).

Diante do exposto é necessária uma mudança radical no controle dos parasitas no Nordeste. O controle preferencialmente químico deve ser substituído pelo controle integrado, incluindo: 1) preparar pastagens ou áreas de caatinga livres ou com baixa contaminação por parasitas na refugia, para utilizar com as categorias mais susceptíveis, como fêmeas no peri-parto e animais jovens. Essas pastagens podem também ser áreas que foram utilizadas com lavouras ou com outras espécies animais (bovinos o equinos); 2) fazer rotação anual dos vermífugos e selecionar drogas antiparasitárias após realização de testes de RA; 3 ) determinar corretamente o peso dos animais a serem dosados evitando a subdose que, além de ser prejudicial por não controlar a doença, no caso de alguns grupos de anti-helmínticos (levamisol e bezimidazóis) pode causar RA; 4) utilizar a dose correta da droga para cada espécie, levando em consideração que, para a maioria dos antihelmínticos, os caprinos necessitam de doses maiores que os ovinos (Torres-Acosta \& Hoste 2008, Costa et al. 2009); 5) restringir o alimento antes do tratamento, isto promove uma redução da motilidade gastrintestinal permitindo uma melhor absorção e maior permanência do medicamento no organismo animal; 6) manejar a lotação dos piquetes evitando a superlotação, pois além de aumentar a contaminação das pastagens, quando os animais pastam até as partes mais baixas do capim, aumenta a taxa de contaminação devido à maior presença de larvas; 7) tratar animais recém adquiridos de forma comprovadamente eficiente (confirmado mediante OPG), evitando a introdução na propriedade de parasitos resistentes; 8) evitar, se possível, soltar os animais nas pastagens nas horas mais frescas do dia, uma vez que nesses horários as larvas infectantes estão na ponta dos capins junto às gotas do orvalho facilitando sua ingestão; 9) colocar animais de diferentes espécies no mesmo pasto pode ser útil, pois as larvas de parasitos de caprinos e ovinos que venham a ser ingeridas por outras espécies poderão ser destruídas no trato gastrintestinal. Lamentavelmente, isto não funciona quando caprinos e ovinos pastoreiam juntos, pois não há especificidade entre os parasitas destas espécies; e 10) manter os animais em bom nível nutricional. (Molento 2005, Vieira 2007, Cézar et al. 2008, Torres-Acosta \& Hoste 2008).

No Brasil e no Nordeste o interesse por anti-helmínticos naturais é crescente e o efeito anti-helmíntico de diversas plantas tem sido testado in vivo e in vitro (Almeida 2005, Furtado 2006), no entanto não há resultados que comprovem a eficiência desses produtos como uma alternativa ao uso de anti-helmínticos, particularmente pela baixa eficiência das plantas testadas e pela falta de identificação de compostos químicos responsáveis por esses efeitos antiparasitários.
Em casos de surtos de parasitoses gastrintestinais é importante recomendar o tratamento e, após uma semana, constatar a eficiência do mesmo mediante exame de McMaster dos animais tratados. Se for constatada baixa eficiência é necessário fazer um teste de RA para, posteriormente, recomendar a droga adequada e as medidas de controle integrado possíveis de serem realizadas na fazenda.

\section{REFERÊNCIAS}

Ahid S.M.M., Suassuna A.C.D., Maia M.B., Costa V.M.M. \& Soares H.S. 2008. Parasitos gastrintestinais em caprinos e ovinos da região oeste do Rio Grande do Norte, Brasil. Ciênc. Anim. Bras. 9(1):212218.

Almeida W.V.F. 2005. Uso de plantas medicinais no controle de helmintos gastrintestinais de caprinos naturalmente Infectados. Dissertação de Mestrado em Zootecnia, Universidade Federal de Campina Grande, Patos, PB. 85p.

Arosemena N.A.E., Bevilaqua C.M.L., Melo A.C.F.L. \& Girão M.D. 1999. Seasonal variations of gastrointestinal nematodes in sheep and goats from semi-arid area in Brazil. Revta Med. Vet. 150:873876.

Bath G.F. \& Van Wyk, J.A. 2001. Using the Famacha system on commercial sheep farms in South Africa. Anais International Sheep Veterinary Congress, Cape Town, South Africa, Vol.1, p.3.

Bath G.F., Hansen J.W., Krecec R.C., Van Wyk J.A. \& Vatta A.F. 2001. Sustainable approaches for managing haemonchosis in sheep and goats. FAO Animal Production and Health Paper, Rome. 89p.

Charles T.P., Pompeu J. \& Miranda D.B. 1989. Efficacy of three broadspectrum anthelmintics against gastrointestinal nematode infections of goats. Vet. Parasitol. 34:71-75.

Cezar A.S., Catto J.B. \& Bianchin I. 2008. Controle alternativo de nematódeos gastrintestinais dos ruminantes: atualidade e perspectivas. Ciência Rural 38(7):2083-2091.

Costa V.M.M., Simões S.V.D. \& Riet-Correa F. 2009. Doenças parasitárias em ruminantes no semi-árido brasileiro. Pesq. Vet. Bras. 9(7): 563-568.

Costa E.A. \& Vieira L.S. 1984. Evolução do parasitismo por nematódeos gastrintestinais em caprinos no sertão dos Inhamus, Ceará. Pesquisa em Andamento oํ 9, Embrapa-CNPCO, Sobral, p.1-4.

Furtado S.K. 2006. Alternativas fitoterápicas para o controle da verminose ovina no estado do Paraná: testes in vitro e in vivo. Tese de Doutorado em Ciências, Curso de Pós-Graduação em Agronomia, Universidade Federal do Paraná, Curitiba. 125p.

Girão E.S., Medeiros L.P. \& Girão R.N. 1992. Ocorrência e distribuição sazonal de helmintos gastrintestinais de caprinos no Município de Teresina, Piauí. Ciência Rural 22(2):197-202.

Girão E.S., Girão R.N. \& Medeiros L.P. 1980. Prevalência e variação estacional de helmintos gastrintestinais de caprinos no município de Valença do Piauí. Circ.Téc.1, Embrapa-CPAMN, Teresina. 5p.

Grupo Mercado Comum (GMC) 1996. Regulamento técnico para registros de produtos antiparasitários de uso veterinário. Decisão № 4/91, Resolução № 11/93. Merscosul, Resolução № 76.

Hoste H., Torres-Acosta J.F.J. \& Aguilar Caballero A.J. 2007. Nutrition-parasite interactions in goats: Is immunoregulation involved in the control of gastrointestinal nematodes? Parasite Immunol. 30:79-88

Martins Filho E. \& Menezes R.C.A.A. 2001. Parasitas gastrintestinais em caprinos (Capra hircus) de uma criação extensiva na microrregião de Curimataú, Estado da Paraíba, Brasil. Revta Bras. Parasitol. Vet. 10(1):41-44

Melo A.C.F.L. Reis I.F, Bevilaqua C.M.L.,Vieira L.S., Echevarria F.A.M. \& Melo L.M. 2003. Nematódeos resistentes a anti-helmínticos em 
rebanhos de ovinos e caprinos do Estado do Ceará, Brasil. Ciência Rural 33(2):339-344.

Molento M.B. 2005. Avanços no diagnóstico e controle das helmintoses em caprinos. I Simpósio Paulista de Caprinocultura (SIMPAC). Multipress, Jaboticabal, p.101-110.

Molento M.B., Tasca C., Ferreira M., Bononi R. \& Stecca E. 2004. Método Famacha como parâmetro clínico individual de infecção por Haemonchus contortus em pequenos ruminantes. Ciencia Rural 34: 1139-1145.

Pereira I.H.O. 1976. Helmintos em caprinos (Capra hircus) no ecossistema Sertão de Pernambuco, Brasil. Tese ICM Universidade Federal do Rio Grande do Sul. 53p.

Reis I.F. 2004. Controle de nematóides gastrintestinais em pequenos ruminantes: método estratégico versus Famacha $\odot$. Dissertação de Mestrado em Ciências Veterinárias, Universidade Estadual do Ceará, Fortaleza. 79p.

Sebrae 2009. Panorama da ovinocaprinocultura no Brasil. Capturado em 17 fev. 2009. Online. Disponível na internet em <http://www.sebrae .com.br>

Silva W.W., Bevilaqua C.M.L. \& Costa A.L. 1998. Natural evolution of gastrointestinal nematodes in goats (Capra hircus) in the semiarid ecosystem of the Paraíba backwoods, northeastern Brazil. Vet. Parasitol. 80:47-52.

Stear M.J., Bairden K., Bishop S.C., Buitkamp J., Duncan J.L., Gettinby G., McKellar Q.A., Park M., Parkins J.J., Reid S.W.J., Strains S. \& Murray M. 1997. The genetic basis of resistance to Ostertagia circumcincta in lambs. Vet. J. 154:111-119.

Torres-Acosta J.F.J. \& Hoste H. 2008. Alternative or improved methods to limit gastro-intestinal parasitism in grazing sheep and goats. Small Rum. Res. 77:159-173.

Urquhart G.M. \& Armour J. 1998. Parasitologia Veterinária, 2ª ed. Editora Guanabara, Rio de Janeiro. 273p.

Van Wyk J.A. \& Bath G.F. 2002. The FAMACHAÓ system for managing haemonchosis in sheep and goats by clinically indentifying individual animals for treatment. Vet. Res. 33:509-529.

Van Wyk J.A. 2001. Refugia - overlooked as perhaps the most potent factor concerning the development of anthemintic resistance. Onderstepoort J. Vet. Res. 68:55-67

Vercruysse J. 1995. Association for the advancement of veterinary parasitology (WAAVP): Second edition of guidelines for evaluating the efficacy of anthelmintics in ruminants (bovine, ovine, caprine). Vet. Parasitol. 58:181-213.

Vieira L.S. 2008. Métodos alternativos de controle de nematóides gastrintestinais em caprinos e ovinos. Revta Ciênc. Tecnol. Agropec. 2:28-31.

Vieira L.S. \& Cavalcante A.C.R. 1999. Resistência anti-helmíntica em rebanhos caprinos no Estado do Ceará. Pesq. Vet. Bras. 19(3/4):99103.

Vieira L.S., Cavalcante A.G.R. \& Ximenes L.J.F. 1997. Epidemiologia e controle das principais parasitoses de caprinos nas regiões semiáridas do Nordeste do Brasil. Circ. Téc., Embrapa Caprinos, Sobral. 49p.

Vieira L.S., Gonçalves P.C., Costa C.A.E. \& Berne M.E.A. 1989. Redução e esterilização de ovos de nematódeos gastrintestinais em caprinos medicados com anti-helmínticos benzimidazóis. Pesq. Agropec. Bras. 24:1255-1265. 